\title{
COUNTABLY ADDITIVE FULL CONDITIONAL PROBABILITIES
}

\author{
THOMAS E. ARMSTRONG
}

(Communicated by R. Daniel Mauldin)

\begin{abstract}
Armstrong-Sudderth showed that a method of Carlson could be adapted to construct examples of countably additive full conditional probabilities. Here it is shown that all countably additive full conditional probabilities arise in this fashion. It is also shown that if the dimensionally ordered family of measures associated by Renyi to a full conditional probability contains an unbounded measure the full conditional probability fails to be countably additive. This leads to the notion of proper full conditional probabilities which if well founded in the sense of Krauss agree with the class of full conditional probabilities arising from Carlson's construction. Section 3 explores the connection between coherence of countable betting systems and countable additivity of full conditional probabilities.
\end{abstract}

\section{INTRODUCTION}

A bookie offers bets in the form of rates of exchange measuring relative likelihood $r(A, B)$ for pairs of events in some class $\mathscr{E}$. Armstrong and Sudderth [3] have given necessary and sufficient conditions on $r$ to ensure that the bookie acts in a locally coherent fashion so that a sure loss on the outcomes involved in a compound bet cannot be inflicted by a gambler on the bookie. These conditions are that the exchange rates can be extended in a locally coherent fashion from $\mathscr{E}$ to all pairs of events, or equivalently that there exists a family $\left\{m_{\alpha} ; \alpha \in \Gamma\right\}$ of $[0, \infty]$-valued measures, dimensionally ordered in the sense of Renyi, so that $r(A, B)=m_{\alpha}(A) / m_{\alpha}(B)$ if $0<m_{\alpha}(B)<\infty$. These requirements may be restated to require a full conditional probability $P(\cdot \cdot)$ of Renyi [12] or Krauss [10] so that $r(A, B)=P(A \mid A \cup B) / P(B \mid A \cup B)$ for all pairs $(A, B)$. (The sense of full conditional probability used here is not that of Renyi [17] but of Dubins [6])

One example of a locally coherent $r$ is defined on a class $\mathscr{E}$ of pairs of Borel sets in $R^{n}$ is as follows. If $A$ and $B$ are finite sets with $B \neq \phi$ then $r(A, B)=|A| /|B|$ where $|\cdot|$ denotes cardinality. If $0 \leq \alpha \leq n$ and $A$ and $B$

Received by the editors July 6, 1987, and in revised form March 8, 1988, and January 5, 1989. 1980 Mathematics Subject Classification (1985 Revision). Primary 28A60, 28A50; Secondary $60 \mathrm{~A} 10,62 \mathrm{~A} 15$.

Key words and phrases. Full conditional probability, countable additivity, coherence. 
have finite measure for $\alpha$-dimensional Hausdorff measure $h_{\alpha}$ with $0<h_{\alpha}(B)$, then $r(A, B)=h_{\alpha}(A) / h_{\alpha}(B)$. In particular, for $\alpha=n, r(A, B)$ is given by the ratio of Lebesgue measures of $A$ and $B$. If $\alpha$ is an integer and $A$ and $B$ are appropriately rectifiable $\alpha$-dimensional surfaces, then $r(A, B)$ is the ratio of $\alpha$ dimensional surface areas. If $\alpha$ is not an integer, $A$ and $B$ may be fractal sets, à la Mandelbrot [11], of Hausdorff dimension $\alpha$ whose relative likelihood is computed as $h_{c}(A) / h_{c}(B)$. Finally, let $\delta$ be a translation invariant probability on the Borel sets and define $r(A, B)$ to be $\delta(A) / \delta(B)$ if $0<\delta(B)<\infty$. This single example yields conditional probabilities between events, including (or closely related to) most used by statisticians. (See Rogers [15] or Falconer [7] for expositions of the topic of Hausdorff measures.)

One question naturally arises in regard to the previous example. Instead of using a purely finitely additive translation invariant probability to define some of the exchange rates in the preceding example, may a locally coherent exchange rate be defined on all pairs of Borel sets which extends that in the example and so that $A \rightarrow r(A, B)$ is countably additive for all $B$ ? This is tantamount to the existence of a countably additive full conditional probability, with certain constraints. It is of interest that though countable additivity was the milieu in which Renyi worked, characterization and not existence was the substance of his work. Krauss, who worked in a finitely additive context, established existence in that context but viewed existence in the countably additive context as a hard problem. Armstrong and Sudderth, based on a technique of Carlson, did establish existence of countably additive full conditional probabilities. It will be shown that if one has a countably additive full conditional probability, then it may be obtained via Carlson's construction. More generally, the proper wellfounded full conditional probabilities of Krauss will be shown to be the class arising from Carlson's construction. It will follow that there is no countably additive full conditional probability consistent with any "truly improper" prior. Given a partially defined locally coherent exchange rate $r$, the best that can be ensured is that there is a locally coherent extension to all pairs of events so that the measures $A \rightarrow r(A, B)$ are countably additive in $A$ for all $B$ originally involved in the definition of $r$. This is investigated, following deFinetti, framing coherence in terms of countable betting systems.

\section{Preliminary}

As an extension of the notions of coherence and conditional coherence that deFinetti first proposed, Armstrong and Sudderth [3] have put forth the notion of local coherence of betting schemes. The motivation was the ability to deal with improper priors as in Hartigan's framework [8]. To a certain extent the connection with improper priors was presaged by Renyi [12].

Following deFinetti, the symbol $A$ denotes both an event and its characteristic function. A rate of exchange $r$ is a $[0, \infty]$-valued function with domain $\mathscr{E}$ in $\mathscr{B} \times[\mathscr{B} \backslash\{\phi\}]$ where $\mathscr{B}$ is an algebra of subsets of a set $X$. (Actually it is well known that any Boolean algebra is isomorphic to an algebra of sets. Many 
results here extend to the more general setting.) A simple exchange is a bet of the form $r(A, B) B-A$, where the bookie pays a gambler $r(A, B)$ (monetary units) if $B \backslash A$ occurs, $r(A, B)-1$ if $A \cap B$ occurs, -1 if $A \backslash B$ occurs, and 0 otherwise. In particular, if $r(A, B)=\infty$ the simple exchange is $\infty$ on $B,-1$ on $A \backslash B$, and 0 otherwise. A compound exchange is obtained by forming a finite linear combination $e(\lambda)=\Sigma \lambda(A, B)[r(A, B) B-A]$ where $\lambda: \mathscr{E} \rightarrow(-\infty, \infty)$ has $\lambda(A, B)=0$ except for finitely many $(A, B) \in \mathscr{E}$. In general, $0 \cdot \infty$ and $\infty-\infty$ are regarded as undefined, and only compound exchanges avoiding their occurrences are allowed. Local incoherence occurs for $e(\lambda)$ if, as an extended real function on $X, e(\lambda)>0$ on $\operatorname{supp}(\lambda)=\bigcup\{A \cup B: \lambda(A, B) \neq 0\}$. A rate of exchange $r$ is locally coherent if no $e(\lambda)$ demonstrates local incoherence and $r$ is locally incoherent otherwise.

An intuition for rates of exchange is that of relative likelihoods or ratios of expected frequencies of the events involved. Local coherence is the requirement that a gambler not be able to generate a sure loss to the bookie on the outcomes actually involved in a given bet.

The major results of Armstrong and Sudderth [3] may be paraphrased as follows:

Extension Theorem. A locally coherent rate of exchange may be extended from its domain to a locally coherent exchange rate with domain $\mathscr{B} \times[\mathscr{B} \backslash\{\phi\}]$.

Characterization Theorem. In order that rate of exchange $r$ on $\mathscr{B} \times[\mathscr{B} \backslash\{\phi\}]$ be locally coherent, it is necessary and sufficient for (a) and (b) to hold.

(a) If $B \neq \phi, m_{B}: A \rightarrow m_{B}(A)=r(A, B)$ is an additive $[0, \infty]$-valued function with $m_{B}(B)=1$.

(b) For all $A, B$, and $C$ in $\mathscr{B}, m_{B}(A) m_{C}(B)=m_{C}(A)$.

Full Conditional Probability Theorem. If $r$ is a locally coherent rate of exchange with domain $\mathscr{B} \times[\mathscr{B} \backslash\{\phi\}]$, then defining $P(A \mid B)=r(A \cap B, B)$ for $A$ and $B \neq \phi$ yields a full conditional probability $P(\cdot \cdot \cdot)$ in that

(a) If $B \neq \phi$, then $A \rightarrow P(A \mid B)$ is a probability measure in $A$ with $P(B \mid B)=1$.

(b) If $A \subset B \subset C$ and $B \neq \phi$, then $P(A \mid B) P(B \mid C)=P(A \mid C)$.

Conversely, given a full conditional probability, one obtains a locally coherent rate of exchange $r$ by setting $r(A, B)=P(A \mid A \cup B) / P(B \mid A \cup B)$ if $P(B \mid A \cup B) \neq 0$ and by setting $r(A, B)=\infty$ otherwise.

A weak order (i.e., transitive, reflexive complete partial order) $\precsim$ called the Renyi order, on events in $\mathscr{B}$ is induced by a full conditional probability $P$ by setting $A \precsim B$ if and only if $P(B \mid A \cup B)>0$. Thus, $A \sim B$ if and only if $0<P(A \mid A \cup B)$ and $0<P(B \mid A \cup B)$ and $A \prec B$ if and only if $P(A \mid A \cup B)=0$ define the symmetric and asymmetric parts of $\precsim$. In terms of the associated rate of exchange $r$ one has $A \sim B$ if and only if $0<r(A, B)<\infty, A \prec B$ if and only if $0=r(A, B)$ and $A \succ B$ if and only if $r(A, B)=\infty$. The interpretation of $A \succ B$ is that $A$ is much more likely than $B$. 
Given two $[0, \infty]$ valued measures on $\left\{\mu_{1}, \mu_{2}\right\}$ on $\mathscr{B}$, Renyi [13] and Krauss [10] set $\mu_{1} \sim \mu_{2}$ if $\mu_{1}=c \mu_{2}$ with $0<c<\infty$ and set $\mu_{1} \prec \mu_{2}$ if the ideal $\mathscr{F}_{\mu_{1}}=\left\{A: \mu_{1}(A)<\infty\right\}$ is contained in the ideal $\mathscr{N}_{\mu_{2}}$ of $\mu_{2}$-negligible sets. Define $\mu_{1} \precsim \mu_{2}$ if and only if $\mu_{1} \prec \mu_{2}$ or $\mu_{1} \sim \mu_{2}$. A family $\left\{\mu_{\alpha}: \alpha \in \Gamma\right\}$ of $[0, \infty]$-valued measures is said to be dimensionally ordered if and only if it is linearly ordered by $\precsim$ and is a complete family in the sense that for any $A \in B \backslash\{\phi\}$ there is an $\alpha \in \Gamma$ so that $0<\mu_{\alpha}(A)<\infty$. If $\left\{\mu_{\alpha}: \alpha \in \Gamma\right\}$ is only weakly ordered by $\precsim$ and is complete, a single-valued selection from the $\sim$ equivalence classes yields a dimensionally ordered family.

Dimensional Ordering Theorem. If $r$ is a locally coherent rate of exchange with full domain, then $\left\{m_{B}: B \in \mathscr{B} \backslash\{\phi\}\right\}$ is complete and weakly ordered, with $B \rightarrow m_{B}$ an order isomorphism for the respective orders $\precsim$. Selecting one $m_{B}$ by selecting one $B$ from each Renyi equivalence class yields a dimensionally ordered family of measures. Conversely, if $\left\{\mu_{\alpha}: \alpha \in \Gamma\right\}$ is a dimensionally ordered family of $[0, \infty]$-valued measures, then $r(A, B)=\mu_{\alpha}(A) / \mu_{\alpha}(B)$ for the $\alpha \in \Gamma$ with $0<\mu_{c x}(B)<\infty$ yields a locally coherent rate of exchange on $\mathscr{B} \times[\mathscr{B} \backslash\{\phi\}]$.

Every measure $m_{B}$ arising in the Dimensional Ordering Theorem is semiproper in that it fails to be $\{0, \infty\}$-valued and so takes on at least one value in $(0, \infty)$. Conversely, if $\left\{\mu_{\alpha}: \alpha \in \Gamma\right\}$ is dimensionally ordered, so is the family obtained by deletion of all non-semiproper members and one obtains the same rate of exchange. By restricting attention to semiproper $[0, \infty]$-valued measures, the correspondence described in this theorem is bijective up to $\sim$ equivalence of the measures in the dimensionally ordered family. As a result, in the following one may assume that all $[0, \infty]$-valued measures occurring are semiproper.

The example previously alluded to (continuing measure, Hausdorff measures, translation invariant probability) is linearly ordered but incomplete. The following shows that it may be embedded in a dimensionally ordered family.

Dimensionally Ordered Extension Theorem. If $\left\{\mu_{\alpha}: \alpha \in \Gamma\right\}$ is a linearly ordered family of $[0, \infty]$-valued measures under $\precsim$, it may be embedded in a dimensionally ordered family $\left\{\mu_{\alpha}: \alpha \in \Gamma\right\}, \Gamma \subset \Gamma^{\prime}$.

The Dimensionally Ordered Extension theorem allows one to conclude the existence of full conditional probabilities agreeing with certain natural conditional probabilities in a wide range of circumstances but does not suffice to yield the existence of any countably additive full conditional probabilities. (As remarked before, existence was not considered by Reyi and was not attacked by Krauss except in the finitely additive case.) The following technique of Carlson allows construction of countably additive full conditional probabilities or exchange rates. The converse to this theorem is the main content of $\S 2$.

Carlson's Construction. Let $\left\{\mu_{\alpha}: \alpha<\alpha_{0}\right\}$ be a complete family of finite measures on $\mathscr{B}$ which has been indexed by the ordinal $\alpha_{0}$. If $r(A, B)$ is $\mu_{r(}(A) /$ $\mu_{r \gamma}(B)$ where $\alpha$ is the first ordinal with $0<\mu_{\alpha x}(A \cup B)$, then $r(\cdot, \cdot)$ is locally 
coherent. For any $A$ and $B \neq \phi$, the corresponding conditional probability $P(A \mid B)$ is $\mu_{\alpha}(A \cap B) / \mu_{c}(B)$, where $\alpha$ is the first ordinal with $0<\mu_{\alpha}(B)$.

\section{COUNTABLE ADDITIVITY OF FULL CONDITIONAL PROBABILITIES} AND EXCHANGE RATES

It is desirable when dealing with a $\sigma$-complete algebra of events to have probabilities and conditional probabilities be countably additive. This section is devoted to the study of the extent to which this is achievable. The goal would be to have a full conditional probability so that $A \rightarrow P(A \mid B)$ is countably additive in $A$ for all $B \neq \phi$. Equivalently, one should have $A \rightarrow m_{B}(A)=r(A, B)$ countably additive in $A$ for some fully defined rate of exchange which extends a partially defined locally coherent rate of exchange given a priori.

Our first result is a negative result, which implies that for our canonical example defined using counting measure and Hausdorff measures, there is no countably additive fully defined locally coherent rate of exchange extending that in the example. The reason, simply stated, is that improperness entails strong finite additivity. The definition of improperness for a $[0, \infty]$ valued measure $m$ will be taken to be that $\sup \{m(A): m(A)<\infty\}=\infty$. Recall that $m$ is semifinite iff $m(A)=\sup \left\{m\left(A^{\prime}\right): A^{\prime} \subset A, m\left(A^{\prime}\right)<\infty\right\}$ for all $A$. For semifinite measures $m(X)=\infty$ iff $m$ is improper. Associated with any measure $m$ is its semifinite part $m^{0}$, which is the largest semifinite minorant defined by $m^{0}(A)=\sup \left\{m\left(A^{\prime}\right): A^{\prime} \subset A, m\left(A^{\prime}\right)<\infty\right\}$ for $A$ in $B$. Thus, $m$ is proper precisely when $m^{0}(X)<\infty$. The definition of strong finite additivity of a $[0, \infty]$-valued measure $m$ will be that there is a disjoint sequence of $m$-null sets $\left\{E_{n}: n \in N\right\}$ so that $X$ is the supremum of $\left\{E_{n}: n \in N\right\}$, Armstrong-Prikry [2]. A slightly weaker definition would be that each set $A$ of finite measure admits a partition by $m$-null sets. The definitions are equivalent if $m$ is finite or $\sigma$-finite.

Let $P$ be a full conditional probability with associated rate of exchange $r$ and dimensionally ordered family $\left\{m_{\alpha}: \alpha \in \Gamma\right\}$ of semiproper measures. $P(\cdot \mid B)$ is countably additive for all $B \neq \phi$ iff $r(\cdot \mid B)$ is countably additive when restricted to an arbitrary set of finite measure for all $B \neq \phi$. This is equivalent to countable additivity of each $m_{\alpha}$ when restricted to sets of finite measure and to the same condition for each $m_{\alpha}^{0}$. Actually, as this proposition shows, one has unrestricted countable additivity.

Proposition 2.1. The following are equivalent:

(a) $P(\cdot \mid B)$ is countably additive if $B \neq \phi$.

(b) $r(\cdot \mid B)$ is countably additive if $B \neq \phi$.

(c) $m_{\alpha}$ is countably additive if $\alpha \in \Gamma$.

(d) $m_{c x}^{0}$ is countably additive. 
Proof. Only (a) $\Rightarrow$ (b) needs proof. It must be shown for a fixed $B \neq \phi$ that if $r\left(A_{n}, B\right) \leq M<\infty$ for $n \in N$ and $A_{n}$ increases to $C$ then $r(C, B)<\infty$. If not, $r(C, B)=\infty$ so $r(B, C)=0$. In this case $P\left(A_{n} \mid C\right)=r\left(A_{n}, C\right)=$ $r\left(A_{n}, B\right) r(B, C)=0$ for $n \in N$. This contradicts countable additivity of $P(\cdot \mid C)$. This establishes that $(\mathrm{a}) \Rightarrow(\mathrm{b})$.

Proposition 2.2. Let $P$ be a full conditional probability on the $\sigma$-complete Boolean algebra $\mathscr{B}$. If $\left\{m_{\alpha}: \alpha \in \Gamma\right\}$ is the associated dimensionally ordered family of semiproper measures and one $m_{\alpha}$ is improper, then $A \rightarrow P(A \mid C)$ is strongly finitely additive for some $\mathscr{C} \in \mathscr{B} \backslash\{\phi\}$.

Proof. Let $\left\{A_{n}: n \in N\right\}$ be a sequence in $\mathscr{B} \backslash\{\phi\}$ so that $\left\{m_{\alpha}\left(A_{n}\right): n \in\right.$ $N\}$ strictly increases to $\infty$. Let $C=\sup \left\{A_{n}: n \in N\right\}$. If it is shown that $P\left(A_{n} \mid C\right)=0$ for all $n$ the proposition will follow. Let $B$ be such that $m_{\alpha}=m_{B}$, and let $r$ be the rate of exchange associated with $P$. We have $0<m_{c x}\left(A_{n}\right) / m_{r}(B)=r\left(A_{n}, B\right) \leq r(C, B) \leq \infty$ for all $n$. Further, $\lim _{n \rightarrow \infty} r\left(A_{n}, B\right)=\infty$ so $r(C, B)=\infty$. As a result $r\left(C, A_{n}\right)=r(C, B) r\left(B, A_{n}\right)$ $=\infty$ for all $n$. Thus $P\left(A_{n} \mid C\right)=r\left(A_{n}, C\right)=0$ for all $n$.

As a result of this proposition, any countably additive full conditional probability $P$ (rate of exchange $r$ ) must be proper in having its associated dimensionally ordered family of semiproper measure consist entirely of proper measures. As a result, all full conditional probabilities arising from Carlson's construction applied to a family of finite countably additive measures are proper. The following proposition shows that this remains true even if the finite measures in the family are not required to be countably additive.

Proposition 2.3. Let $\left\{\mu_{\gamma}: \gamma<\alpha_{0}\right\}$ be a complete family of finite measures where $\alpha_{0}$ is an ordinal. Carry out Carlson's Construction to get a full conditional probability $P(\cdot \cdot \cdot)$, a locally coherent rate of exchange $r$, and an associated dimensionally ordered family $\left\{m_{\alpha}: \alpha \in \Gamma\right\}$ of semiproper measures. For $\alpha \in \Gamma$ let $\mathscr{N}_{\alpha}=\mathscr{N}_{\mu_{n}}$.

(a) Each $m_{c x}$ is proper.

(b) $\Gamma$ is reverse well ordered.

(c) Let $\gamma$ be an ordinal and $\nu_{\gamma}$ be the restriction of $\mu_{\gamma}$, to $\bigcap\left\{\mathscr{N}_{\alpha}: \alpha<\gamma\right\}=$

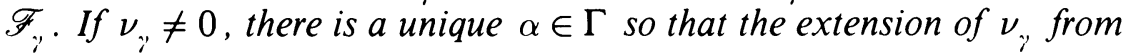
$\mathscr{F}$, to $\mathscr{B}$ equal to $\infty$ on $\mathscr{B} \backslash \mathscr{F}$ is a multiple of $m_{c \gamma}$. The correspondence $\gamma \rightarrow \alpha$ is an order anti-isomorphism from $\left\{\gamma<\alpha_{0}: \nu_{\gamma} \neq 0\right\}$ to $\Gamma$.

Proof. (a) and (b) are consequences of (c).

(c) Let $A \in \mathscr{B} \backslash\{\phi\}$ and let $\alpha(A)$ be the first ordinal with $0<\mu_{\text {r }}(A)$. We have $\mu_{\beta}(A)=0$ so $\nu_{\beta}(A)=0$ if $\beta<\alpha(A)$ hence $A \in \mathscr{F}_{(r(A)}(A)$. As a result for $B \in \mathscr{B}$,

$$
P(B \mid A)=\mu_{r(A)}(A \cap B) / \mu_{r(A)}(A)=\nu_{r(A)}(A \cap B) / \nu_{r(x)}(A) .
$$


As a result,

$$
m_{A}(B)=r(B, A)= \begin{cases}\nu_{\alpha(A \cup B)}(B) / \nu_{\alpha(A \cup B)}(A) & \text { if } \nu_{\alpha(A \cup B)}(A) \neq 0 \\ \infty & \text { else }\end{cases}
$$

If it is the case that $m_{A}(B)=\infty$, then $\alpha(A \cup B)<\alpha(A)$ and $B \in \mathscr{B} \backslash \mathscr{F}_{\alpha(A)}$. If $B \in \mathscr{F}_{\alpha(A)}$, then $m_{A}(B)=\nu_{\alpha(A)}(B) / \nu_{\alpha(A)}(A)$. Thus, if $\nu_{\alpha(A)}$ is extended to be $\infty$ on $\mathscr{B} \backslash \mathscr{F}_{\alpha(A)}$, then $\nu_{\alpha(A)} \sim m_{A}$.

The only $\gamma<\alpha_{0}$ not of the form $\alpha(A)$ for some $A \in B$ are those where $\nu_{\gamma}=0$. This is sufficient to establish that $r \rightarrow \alpha$ is an anti-isomorphism as stated.

Proposition 2.4. Let $P$ be a full conditional probability on the $\sigma$-complete algebra $\mathscr{B}$. If there is a sequence $\left\{A_{n}: n \in N\right\}$ so that $A_{n} \prec A_{n+1}$ for all $n$ ( $\prec$ the strict Renyi order), then $P(\cdot \mid C)$ is strongly finitely additive for some $C$.

Proof. If so, then it may be assumed that $A_{n} \subset A_{n+1}$ for all $n$. In this case a repetition of the argument of Proposition 2.2 shows that $P(\cdot \mid C)$ is strongly finitely additive for $C=\bigcup_{n=1}^{\infty} A_{n}$.

Proposition 2.5. Let $P(\cdot \mid \cdot)$ be a countably additive Renyi full conditional probability on the $\sigma$-complete algebra $\mathscr{B}$. The associated dimensionally ordered family $\left\{m_{\alpha}: \alpha \in \Gamma\right\}$ of proper countably additive measure is reverse well ordered. If $\alpha_{0}$ is the ordinal anti-isomorphic to $\Gamma$ with $\gamma \rightarrow \alpha(\gamma)$, the anti-isomorphism from $\alpha_{0}$ to $\Gamma$, then $P(\cdot \mid \cdot)$ is the full conditional probability given by Carlson's construction based on $\left\{m_{\alpha(\gamma)}: \gamma<\alpha_{0}\right\}$.

Proof. From Proposition 2.4 it follows that there is no strictly increasing sequence in $\mathscr{B}$ for the Renyi order. Thus, $\Gamma$ contains no strictly increasing sequence. As a result, $\Gamma$ is reverse well ordered. Proposition 2.3 applied to $\left\{m_{\alpha(\gamma)}: \gamma<\alpha_{0}\right\}$ suffices to finish the demonstration of this proposition.

It should be remarked that $\sigma$-complete algebras have been used as the natural milieu for countably additive measures. It need not be the case that a $\sigma$-complete algebra admit a complete family of countably additive measures (e.g. the regular open sets in $[0,1])$. On the other hand, countable additivity is a well-defined notion for measures even if the algebra is not $\sigma$-complete. There do exist infinite algebras with every finitely additive probability countably additive. These are precisely those in which every strictly increasing sequence fails to have a supremum (see Armstrong [1]). For such algebras Propositions 2.2 and 2.4 are invalid.

Krauss [10] calls a full conditional probability well founded if there is no sequence of sets strictly increasing for the Renyi order (i.e. the Renyi order is well founded). As a result, infinite algebras exist with all full conditional probabilities countably additive but with some not well founded. This contradicts the conclusions of Propositions 2.4 and 2.5. Such full conditional probabilities cannot arise via Carlson's construction, for his procedure only gives rise to wellfounded full conditional probabilities. In fact, we have the following converse to Proposition 2.3. 
Proposition 2.6. If $P$ is a proper, well founded full conditional probability on $\mathscr{B}$, then it may be obtained via Carlson's construction.

Proof. A simple adaptation of the proof of Proposition 2.5.

\section{3. $\sigma$-LOCAL COHERENCE}

In [5] deFinetti discusses countable additivity in some detail. His view is that of sequential continuity so that if a sequence $\left\{A_{n}\right\}$ of events (in a $\sigma$-algebra of sets) converges pointwise to $A$, then $P\left(A_{n}\right) \rightarrow P(A)$. Similarly for conditional probabilities, when in addition a sequence $\left\{B_{n}\right\}$ of conditioning events is converging to $B$, then $P\left(A_{n} \mid B_{n}\right) \rightarrow P(A \mid B)$. Only briefly [5, $\left.\S 5.20\right]$ is the topic of coherence of countable betting systems, the main topic to be addressed here, taken up. This is dismissed with the remark "But in reality the argument is circular, for only if we know that complete additivity holds can we think of extending the notion of combinations of fair bets to combinations of an infinite number of bets with the corresponding sequence of betting odds." On the contrary, one may easily evaluate the combined bet as an extended real-valued function without reference to any measure if pointwise convergent. The natural question when presented with a probability measure, usually given by ordinary coherence, is whether expectations of the finite partial sums of bets converge to that of the infinite sum of bets. When the fair bets are simple exchanges with respect to a locally coherent rate of exchange, the expected value of all finite partial sums should be zero. Violations of this will lead to $\sigma$-incoherence (as defined below) of the rate of exchange and to the converse. Heath and Sudderth [9] have studied countable additivity and coherence. The material in this section may be considered extensions of their results from probabilities to conditional probabilities.

By countable compound exchanges with respect to a rate of exchange $r$, we mean

$$
e(\lambda)=\sum \lambda(A, B)[r(A, B) B-A]
$$

where for at most countably many pairs $(A, B)$ is $\lambda(A, B) \neq 0$. A finite partial exchange for $\lambda$ is one obtained from $\lambda$ by setting all but finitely many $\lambda(A, B)$ which are nonzero equal to zero. An exchange is boundedly pointwise convergent if its finite partial exchanges converge pointwise and are uniformly bounded. Any boundedly pointwise convergent exchange $e(\lambda)$ is unambiguously defined as a bounded function. Adopting pointwise sequential convergence of exchanges dictates that $\mathscr{B}$ should be a $\sigma$-algebra. A rate of exchange $r$ will be said to be $\sigma$-locally incoherent if there is a boundedly pointwise convergent exchange $e(\lambda)$ with $e(\lambda)>0$ on $\operatorname{supp}(\lambda)=\bigcup\{A \cup B: \lambda(A, B) \neq \phi\}$. Otherwise, $r$ is $\sigma$-locally coherent. If $r$ is $\sigma$-locally coherent, then it is locally coherent. (Analogous definitions of $\sigma$-conditional coherence and $\sigma$-coherence could be made in the appropriate betting contexts and would be interpretable as special cases of $\sigma$ local coherence). 
Proposition 3.1. Let $r$ be a fully defined locally coherent rate of exchange. Let $B$ be in $\mathscr{B} \backslash\{\phi\}$ with $m_{B}: A \rightarrow r(A, B)$ and let $m_{B}^{0}$ be the semifinite part of $m_{B}$.

(a) The following are equivalent:

(1) $m_{B}^{0}$ is countably additive.

(2) For no boundedly pointwise convergent exchange $e(\lambda)$ with $\operatorname{supp}(\lambda) \sim B$ is it the case that $e(\lambda)>0$ on $\operatorname{supp}(\lambda)$.

(3) For no boundedly pointwise convergent exchange $e(\lambda)$ with $\operatorname{supp}(\lambda) \sim B$ is it the case that $e(\lambda) \geq \epsilon$ on $\operatorname{supp}(\lambda)$ for some $\epsilon>0$.

(b) The following are equivalent:

(1) $\mathscr{F}_{B}=\{A: A \precsim B\}=\left\{A: r(A, B)\right.$ is a $\sigma$-ideal and $m_{B}$ is proper and countably additive.

(2) For no boundedly pointwise convergent exchange $e(\lambda)$ with $B \precsim \operatorname{supp}(\lambda)$ and $\operatorname{supp}\left(\lambda^{\prime}\right) \precsim B$ for all finite partial exchanges $e\left(\lambda^{\prime}\right)$ is it the case that $e(\lambda)>0$ on $\operatorname{supp}(\lambda)$.

(3) For no exchange $e(\lambda)$ as in (2) is there an $\epsilon>0$ with $e(\lambda) \geq \epsilon$ on $\operatorname{supp}(\lambda)$.

Proof. (a) If (1) holds, the bounded convergence theorem implies that $0=$ $\int e(\lambda) d m_{B}^{0}$ since $m_{B}^{0}(\operatorname{supp}(\lambda))<\infty$. If $e(\lambda)>0$ on $\operatorname{supp}(\lambda)$ and $m_{B}^{0}(\operatorname{supp}(\lambda))>$ 0 , it would have to be the case that $0<\int e(\lambda) d m_{B}^{0}$. As a result (2) holds.

(2) implies (3) immediately.

If (3) holds but $m_{B}^{0}$ is not countably additive there is a disjoint sequence $\left\{A_{n}\right\}$ with union $A$ so that $m_{B}^{0}(A)>\sum_{n=1}^{\infty} m_{B}^{0}\left(A_{N}\right)=\lambda$. It may be assumed that $m_{B}^{0}(A)<\infty$. Otherwise, by semifiniteness of $m_{B}^{0}$, there is an $A^{*} \subset A$ with $m_{B}^{0}\left(A^{*}\right)>\lambda$. Replace $A$ with $A^{*}$ and $\left\{A_{n}\right\}$ with $\left\{A_{n} \cap A^{*}\right\}$ to get the desired assumption. Since $m_{B}(A)=m_{B}^{0}(A)$ and $m_{B}\left(A_{n}\right)=m_{B}^{0}\left(A_{n}\right)$ in this case we have $r(A, B)>\sum_{n=1}^{\infty} r\left(A_{n}, B\right)$ and as a result $r(A, B \cup A)=r(A, B) r(B, B \cup A) \geq \sum_{n=1}^{\infty} r\left(A_{n}, B \cup A\right)=\left[\sum_{n=1}^{\infty} r\left(A_{n}, B\right)\right] r(B, B \cup A)$.

Form the exchange

$$
e(\lambda)=[r(A, B \cup A)(B \cup A)-A]-\sum_{n=1}^{\infty}\left[r\left(A_{n}, B \cup A\right)(B \cup A)-A_{n}\right],
$$

which exceeds $r(A, B \cup A)-\sum_{n=1}^{\infty} r\left(A_{n}, B \cup A\right)>0$ on $B \cup A=\operatorname{supp}(\lambda) \sim B$. This contradicts (3), so $m_{B}^{0}$ must be countably additive. Thus, $(3) \rightarrow(1)$.

(b) (2) immediately implies (3).

$(3) \rightarrow(1)$ : Notice that (3) implies condition (3) of (a) so $m_{B}^{0}$ is countably additive. Since $m_{B}^{0}$ and $m_{B}$ agree on $\mathscr{F}_{B}=\left\{m_{B}<\infty\right\}$, countable additivity for $m_{B}$ can fail only if there are disjoint $\left\{A_{n}: n \in N\right\} \subset \mathscr{F}_{B}$ with union $A$ so 
that $m_{B}(A)=\infty>\sum_{n=1}^{\infty} m_{B}\left(A_{n}\right)$. It may be assumed that $B \subset A$. We have $r(A, B)=\infty$, so $r\left(A_{n}, A\right)=0$ for all $n$. Set

$$
e(\lambda)=\sum_{n=1}^{\infty} A_{n}=\sum_{n=1}^{\infty}(-1)\left[r\left(A_{n}, A\right) A-A_{n}\right]=A
$$

to obtain a contradiction of $(3)$, in this case since $\left(A_{n} \precsim B \prec A\right)$. Thus, $m_{B}$ is countably additive. At the same time, this argument shows that if $\left\{A_{n}: n \in\right.$ $N\} \subset \mathscr{F}_{B}$ are disjoint, then $m\left(\bigcup_{n=1}^{\infty} A_{n}\right)<\infty$ so $\bigcup_{n=1} A_{n} \in \mathscr{F}_{B}$. As a result, $\mathscr{F}_{B}$ is a $\sigma$-ideal. Since $m_{B}$ is real valued on $\mathscr{F}_{B}$ and $\mathscr{F}_{B}$ is a $\sigma$-ideal, $m_{B}$ is bounded on $\mathscr{F}_{B}$. The latter statement is equivalent to properness of $m_{B}$.

$(1) \rightarrow(2)$ : Let $e(\lambda)$ be a boundedly pointwise convergent exchange with $\operatorname{supp}(\lambda) \succsim B$ and with $\operatorname{supp}\left(\lambda^{\prime}\right) \precsim B$ for all partial exchanges $e\left(\lambda^{\prime}\right)$. Each $e\left(\lambda^{\prime}\right)$ is $m_{B}^{0}$ integrable and $0 \leq m_{B}^{0}(\operatorname{supp}(\lambda))<\infty$ since $m_{B}^{0}$ is finite. The bounded convergence theorem implies that $0=\int e(\lambda) d m_{B}^{0}=\int_{\operatorname{supp}(\lambda)} e(\lambda) d m_{B}^{0}$. If this is the case, it is impossible that $e(\lambda)>0$ on $\operatorname{supp}(\lambda)$.

As a corollary we have this proposition.

Proposition 3.2. For a fully defined locally coherent exchange rate $r$ to be countably additive it is necessary and sufficient that it be $\sigma$-locally coherent.

One open question is whether a strengthening of $\sigma$-local coherence as in (2) of (b) of Proposition 3.1 is necessary and sufficient so that a partially defined rate of exchange admits an extension to a fully defined countably additive rate of exchange.

\section{REFERENCES}

1. T. E. Armstrong, When do the regular sets for a finitely additive Borel measure form a $\sigma$ algebra?, J. Austral. Math. Soc. Ser. A 33 (1982), 374-385.

2. T. E. Armstrong and K. Prikry, $\kappa$-additivity and $\kappa$-finiteness of measures on sets and left invariant measures on discrete groups, Proc. Amer. Math. Soc. 80 (1980), 105-112.

3. T. E. Armstrong and W. Sudderth, Locally coherent rates of exchange, Annals of Statistics 17, (1989) (to appear).

4. T. Carlson, A solution of Ulam's problem on relative measure, Proc. Amer. Math. Soc. 94 (1985), 129-134.

5. B. deFinetti, Probability, induction, and statistics, Wiley, New York, 1972.

6. L. E. Dubins, Finitely additive conditional probabilities, conglomerability and disintegrations, Ann. Probab. 3 (1975), 89-99.

7. H. J. Falconer, The geometry of fractal sets, Cambridge University Press, Cambridge, 1985.

8. J. Hartigan, Bayes theory, Springer-Verlag, Berlin-New York, 1983.

9. D. Heath and W. Sudderth, On finitely additive priors, coherence, and extended admissibility, Ann. Statist. 6 (1978), 333-345.

10. P. H. Krauss, Representation of conditional probability measures on Boolean algebras, Acta Math. Hungar. 19 (1968), 229-241.

11. B. B. Mandelbrot, Fractals: form, chance and dimension, Freeman, San Francisco, 1977.

12. A. Renyi, On a new axiomatic theory of probability, Acta Math. Hungar. 6 (1955), 285-355. 
13. - On conditional probability spaces generated by a dimensionally ordered set of measures, Theory Probab. Appl. 1 (1956), 61-71.

14. __ Foundations of probability, Holden-Day, San Francisco, 1970.

15. C. A. Rogers, Hausdorff measures, Cambridge University Press, Cambridge, 1970.

Department of Mathematics and Statistics, University of Maryland-Baltimore County, Catonsville, Maryland, 21228 